within each time point block and between the time point blocks using linear mixed effect models with random intercept by the subject.

Results: There was significant increase in SPI after positioning the patient in prone position $(p \leq 0.001)$. There was no significant change in MAP and HR but PPV showed significant change when patients were positioned prone ( $p=0.001)$. Moderate correlation was noted between SPI and MAP. Negative correlation was noted between SPI and PPV.

Conclusions: SPI changes when position is changed from supine to prone. Therefore, interpretation of SPI as a surrogate measure of nociception-antinociception balance could therefore be confounded in various situations.

\section{A017 Bronchoscopy-Guided Percutaneous Dilatational Tracheostomy in Critically III Patients: A Study of Complication and Expanded Utility}

Suprith C., ${ }^{1}$ Madhusudan Kalluraya, ${ }^{1}$ Deepak Ramachandra, ${ }^{1}$ Mehta R. ${ }^{1}$

${ }^{1}$ Critical Care Medicine, Apollo Speciality Hospital, Jayanagar, Bengaluru, Karnataka, India

Background: Bronchoscopy-guided percutaneous dilatational tracheostomy (BG-PDT) is one of the most frequently performed procedures in critically ill patients. Complications occur in $5 \%$ to $40 \%$ of tracheostomies depending on study design, patient follow-up, and the definition of the different complications. The mortality rate of PCT is less than $2 \%$. We conducted this study to assess the complications and expanded utility of BG-PDT in the Indian setting.

Materials and Methods: A retrospective analysis of 116 BG-PDT performed in the past 3 years was done. PDT was done using the standard technique, using the Cook PDT kit and videobronchoscopy guidance, with all steps done under vision. Postprocedure, all clots which migrated to the distal airway were suctioned. Complications were noted. Additional BAL was done in patients with infiltrates for cultures.

Results: One hundred sixteen BG-PDT were performed. The most common indication for BG-PDT was anticipated prolonged ventilator stay to facilitate weaning. Complications were divided into intra-procedural and early postprocedural complications. Positive BAL culture was also analyzed.

Conclusions: Our study shows that BG-PDT is safe with minimal complications, when performed by experienced ICU personnel. The advantages include confirming all steps under vision, postprocedure suctioning of clots, and BAL done in the same setting. We propose BG-PDT as an "expanded PDT strategy," combining a safe visually guiding procedure with clot clearance, and additional BAL for cultures.

\section{A018 Observational Study of Intracranial Pressure Variation Using Ultrasonography Guided Optic Nerve Sheath Diameter in Trendelenburg Position among Major Abdominal Laparoscopic Surgery \\ Shobha Purohit, ${ }^{1}$ Gaurav Sharma, ${ }^{1}$ Deeksha Singh ${ }^{1}$ \\ ${ }^{1}$ Department of Anaesthesiology, SMS Medical College and \\ Hospital, Jaipur, Rajasthan, India}

Background: Laparoscopic surgeries are widely established due to its various benefits. It requires adequate surgical exposure that is achieved by pneumoperitoneum often combined with Trendelenburg position that collectively causes increase in intra-abdominal pressure leading to many systemic physiological consequences including increase in intracranial pressure. Noninvasive USG guided ONSD was used as their measurement correlates with invasive method in patients of neurocritical care unit. We aimed to investigate the effect of Trendelenburg position with pneumoperitoneum on ONSD to examine possible changes in ICP in patients undergoing major laparoscopic abdominal surgery.

Materials and Methods: Study included 40 patients of ASA I, II with no neurological disease undergoing major abdominal laparoscopic surgery. USG guided ONSD was measured in both eyes $3 \mathrm{~mm}$ behind the globe preoperatively, following induction, 3 min after Trendelenburg position and 3 min with pneumoperitoneum, 2 minutes after desufflation; other parameters observed were HR, SBP, DBP, MAP, Ppeak, and ETCO2.

Results: ONSD was significantly increased from baseline in Trendelenburg position $(p<0.001)$. Mean ONSD at Trendelenburg position $(5.2 \pm 0.8)$ was significantly more than that in supine position $(4.7 \pm 0.7)$. Four patients had ONSD > $5.8 \mathrm{~mm}$ (the cutoff value for prediction of ICP above $20 \mathrm{~mm} \mathrm{Hg}$ in previous studies). Mean ONSD at Trendelenburg with pneumoperitoneum (5.6 \pm 0.8$)$ increased compared with supine position. Seventeen patients had ONSD > $5.8 \mathrm{~mm}$.

Conclusions: We conclude that ONSD which reflects ICP was significantly increased from baseline in Trendelenburg's position with pneumoperitoneum.

\section{A019 Hydrocephalus Secondary to Cervical Spinal Cord Surgery \\ Subhajit Guha, ${ }^{1}$ Indranil Ghosh, ${ }^{1}$ Bibhukalyani Das ${ }^{1}$ \\ ${ }^{1}$ Department of Neurocritical Care, Institute of Neurosciences Kolkata (INK), Kolkata, West Bengal, India}

Background: Cerebrospinal fluid (CSF) leakage is a common complication after spinal tumor resection that resolves naturally in many cases. Hydrocephalus with CSF leakage as a complication after spinal surgery is rare. Here, we report a rare case of delayed hydrocephalus due to CSF leakage after cervical cord tumor surgery.

Case Description: A 60-year-old lady with gait disturbance and numbness of both hands underwent uneventful surgery of C1-C2 partial laminectomy and excision of schwannoma. Three months after surgery she was readmitted with features of postoperative meningitis. CSF examination was normal while CT scan showed very small pseudomeningocele. She improved with antipyretics and empirical antibiotics. Two months after this episode she presented in a deeply comatose state. CT brain revealed gross hydrocephalus and she became conscious only after VP shunt.

Conclusion: Hydrocephalus due to CSF leakage is a rare complication after spinal surgery. In our case, CSF did not prove septic meningitis. However, inflammation may 
have been caused by aseptic meningitis, and microhemorrhage and fibrinogenic components might have been causes of CSF circulation disorder and absorption disorder in the subarachnoid space. This situation may have then led to delayed hydrocephalus. This is a rare case of delayed hydrocephalus after CSF leakage following spine tumor surgery.

\section{A020 Asleep-Awake-Asleep Technique with Endotracheal General Anesthesia in a Patient of Hemiballismus for Pallidotomy: A Unique Challenge \\ Ninad Dhokte, ${ }^{1}$ Ratan Chelani ${ }^{1}$ \\ ${ }^{1}$ Department of Anaesthesia, P. D. Hinduja Hospital, Mumbai, Maharashtra, India}

Background: Hemiballismus is a relatively rare movement disorder. It is usually caused by a lesion that involves the contralateral subthalamic nucleus (STN). Stereotactic pallidotomy is the procedure of choice in the treatment of medically intractable hemiballismus.

Case Description: A 24-year-old female with history of left thalamic and midbrain cavernoma having right sided hemiballistic movements with right sided weakness, underwent craniotomy and excision of cavernoma under general anesthesia in February 2019. Patient got symptomatic relief from hemiballismus for 2 months, again developed hemiballismus of increased severity in spite of pharmacological treatment. Hence, it was decided to go for surgical treatment in form of left pallidotomy. After all preoperative workup, patient was given general anesthesia with standard doses of inducing agent propofol and muscle relaxant atracurium and maintained on sevoflurane. Patient was then taken to MRI and CT scan for mapping of globus pallidus. Patient was shifted to operating room and left sided burr hole was completed under anesthesia. Stereotactically, left-sided pallidus was identified. Wakeup test was done and patient was asked to follow the commands for checking effects of left pallidotomy. After confirming benefits with help of neurologist, permanent pallidotomy was done. Effects of pallidotomy rechecked and patient was taken under general anesthesia again, shifted to MRI for confirmation of lesion of pallidotomy. Patient was then reversed and extubated in operating room.

Conclusion: Pallidotomy presents unique challenge to attending anesthesiologist. Anesthesia was given by asleep-awake-asleep technique. Dexmedetomidine and narcotic based anesthesia was the technique of choice.

\section{A021 Airway Management in Children with Noma Sequelae Undergoing Craniomaxillofacial Reconstructive Surgery \\ Wesley Rajaleelan, ${ }^{1}$ Marloes Otterman ${ }^{2}$ \\ 'Department of Anesthesia, Toronto Western Hospital, University Health Network, Toronto, Canada \\ ${ }^{2}$ Department of Anesthesia, University Medical Centre, Utrecht, The Netherlands}

Background: Noma (cancrum oris) is an exclusive disease of childhood characterized by ulcerative necrosis of the maxillofacial structures, affecting up to $1,40,000$ children annually. It is fatal in 80 to $90 \%$ of cases in the acute setting. Survivors are left with disfiguring maxillofacial deformations that make airway manipulation for reconstructive surgery very challenging.

Materials and Methods: Sixteen patients with chronic sequelae of noma, underwent maxillofacial reconstructive surgery at the Noma Hospital for Children, Sokoto, Nigeria. The procedures were done by Medicines Sans Frontiers (OCA) mission. The Each patient posed significant airway challenges due to anatomic malformations, trismus, and restricted neck movements. Lack of preoperative imaging and limited resources added to the challenge. We were able to surmount these with the use of a three-tier hierarchical plan: plan A (intended airway management strategy), plan B (secondary management strategy), and plan C (surgical access to the trachea).

Results: Preoperative workup included measuring thyromental, sternomental, and inters incisor distances, neck movements, and mouth opening. Of the 16 patients in this series, 14 were intubated using plan A. Two required deployment of plan B and none required plan C. We predominantly used fiber optic and nasal intubation for these patients.

Conclusions: Maxillofacial reconstructive surgery for Noma poses a huge challenge to the anesthesiologists, especially in children. Adequate planning, screening, and assessment of the airway with primary, secondary, and back-up plans are crucial. With this strategy in place "cannot intubate, cannot ventilate" situations can be handled during an emergency. Psychological and nutritional rehabilitation is essential prior to surgery.

A022 Hematuria during Lumbar Spine Surgery in Prone Position: A Perioperative Challenge

Saurav Singh, ${ }^{1}$ Priyanka Gupta, ${ }^{1}$ Ashutosh Kaushal, ${ }^{1}$ Konish Bishwas'

${ }^{1}$ Department of Anaesthesiology, All India Institute of Medical Sciences, Rishikesh, Uttarakhand, India

Background: Hematuria in patients undergoing surgery in prone position is a perioperative diagnostic challenge, making it difficult to find the source of bleeding in this position. Gross hematuria during this period adds to the surgical blood loss, contributing to morbidity, and mortality. We present a case of intraoperative hematuria during lumbar spine surgery in prone position which was investigated postoperatively.

Case Description: A 35-year-old female patient of ASA class 1 suffered a fall from fourth floor of construction building with impact on her feet. Radiological assessment at the time of admission revealed L1 burst fracture. Transforaminal lumbar fixation was planned on fourth day following injury. Patient was induced and positioned prone. Thirty minutes after commencement of surgery, sudden onset of frank hematuria was noted which continued over 10 minutes, amounting to approximately $400 \mathrm{~mL}$. Surgery was stopped. Following urosurgical consultation, catheter was 